\title{
Functionalization of Wool with L-Cysteine: Process Characterization and Assessment of Antimicrobial Activity and Cytotoxicity
}

\author{
Isabel C. Gouveia, ${ }^{1,2}$ Daniela Sá, ${ }^{3}$ Mariana Henriques ${ }^{4}$ \\ ${ }^{1}$ Textile Department, University of Beira Interior, 6201-001Covilhã-Portugal \\ ${ }^{2} R \mathcal{E} D$ Unit of Textile and Paper Materials, University of Beira Interior, 6201-001Covilhã-Portugal \\ ${ }^{3}$ Chemistry Department, University of Beira Interior, 6201-001Covilha-Portugal \\ ${ }^{4}$ Institute for Biotechnology and Bioengineering, Centre of Biological Engineering, University of Minho, \\ Campus de Gualtar, 4710-057 Braga, Portugal
}

Received 19 April 2010; accepted 1 February 2011

DOI 10.1002/app.34587

Published online 18 October 2011 in Wiley Online Library (wileyonlinelibrary.com).

\begin{abstract}
This investigation reports a new biotechnological process that uses L-cysteine (L-Cys) which provides a permanent, nontoxic and effective antimicrobial effect over wool-based materials. This process is simple and carried out via widespread exhausting methods. Typically, wool fabrics are incubated with L-Cys for $50 \mathrm{~min}$ at $60^{\circ} \mathrm{C}$ in a $\mathrm{pH} 4.8$ acetate buffer solution $25 \mathrm{mM}$, under mild agitation to give a good absorption rate. The minimal inhibitory concentration (MIC) of L-Cys was evaluated by the NCCLS M07-A6 standard method, and the results showed a good antibacterial activity against $S$. aureus and $K$. pneumoniae within the range of $6.0 \times 10^{-3}-4.8 \times 10^{-2} \mathrm{~g} / \mathrm{mL}$ [MIC $\left.0.6 \%(\mathrm{w} / \mathrm{v})\right]$ and 6.0 $\times 10^{-3}-4.8 \times 10^{-2} \mathrm{~g} / \mathrm{mL}$ [MIC $0.6 \%(\mathrm{w} / \mathrm{v})$ ], respectively. In addition, the antimicrobial activity of the functionalized wool was assessed by the international standard JIS 1902-
\end{abstract}

2002 showing a good inhibition of bacterial growth for an L-Cys concentration of $1 \%$ over the weight of fabric, both against Staphylococcus aureus and Klebsiella pneumoniae. Moreover, the biocidal mechanism was found to be related with the increase of sulfhydryl's groups onto wool fibers, which were quantified by the Ellman's reagent (5,5'-Dithio-bis(2nitrobenzoic acid) method. The new process is easy to perform, non toxic, preserve wool quality and is a novel biomimetic approach that uses antimicrobial amino acids and may open new avenues for the design of biomedical textiles with a broad range of applications in healthcare. () 2011 Wiley Periodicals, Inc. J Appl Polym Sci 124: 1352-1358, 2012

Key words: antimicrobial textiles; biopolymers; S. aureus; K. pneumoniae; sulfhydryl groups; Ellman's reagent

\section{INTRODUCTION}

Antimicrobial textiles are frequently used in medical/hospital, pediatric, and geriatric fields. In the past, these products were processed and treated using chemicals that would often yield highly toxic or if not undesirable by-products. Moreover, some of these processes and products may alter or even damage the fundamental properties of the component fibers which may cause a product to be less effective in its intended use. Consequently, there have been constant efforts to develop more ecological, effective, and property-preserving processes. ${ }^{1-5}$

Several methods have been developed to give antimicrobial properties to textile materials. ${ }^{1-7}$ In

\footnotetext{
Correspondence to: I. C. Gouveia (igouveia@ubi.pt).

Contract grant sponsor: FCT; contract grant number: PTDC/EBB-BIO/113671/2009.

Contract grant sponsor: $\mathrm{R}$ and D Unit of Textile and Paper Materials, Faculty of Engineering, University of Beira Interior.

Journal of Applied Polymer Science, Vol. 124, 1352-1358 (2012) (C) 2011 Wiley Periodicals, Inc.
}

addition, various antimicrobial agents have been tested in textiles. Quaternary ammonium compounds, silver, polyhexamethylene biguanides, and triclosan have been used with success. They have powerful bactericidal activity as indicated by the MIC values (minimal inhibitory concentration). However, the majority have a reduced spectrum of microbial inhibition and may cause skin irritation, ecotoxicity, and bacterial resistance, and very few processes have been developed toward wool-based materials. ${ }^{1-7}$ Moreover, their incorporation in the textiles reduces the bioactivity substantially and limits availability, compelling the raised concentrations to be used in the functionalization process. Likewise, the biocide can gradually lose activity during the use and launderings of the textile. Thus, great amounts of these biocides are applied to the textiles to control the bacterial growth efficiently and to keep its durability. ${ }^{2,3}$

To overcome these disadvantages, natural compounds mostly chitosan and plant extracts are being tested as antimicrobial agents for textiles. ${ }^{2,4,5}$ Also, the requirement of the consumers for hygienic clothes and the growing market of medical and 
TABLE I

Characterization of the Textile Material

\begin{tabular}{lcc}
\hline \multicolumn{1}{c}{ Characteristic } & $\begin{array}{c}\text { Standard } \\
\text { method }\end{array}$ & \multicolumn{1}{c}{ Value } \\
\hline Composition & AATCC 20 & $100 \%$ Wool fiber \\
Square mass $\left(\mathrm{g} / \mathrm{m}^{2}\right)$ & ISO 3801 & $170 \mathrm{~g} / \mathrm{m}^{2}$ \\
Density & ISO 7211-2 & Warp 21.7 yarns $/ \mathrm{cm}$ \\
& & Weft 19.0 yarns $/ \mathrm{cm}$ \\
$\begin{array}{l}\text { Resistance to } \\
\text { abrasion }\end{array}$ & EN IS0 12947-2 & $34,000 \pm 3,000$ cycles \\
\hline
\end{tabular}

functional textiles stimulated the research and development of new antimicrobial textiles.

In view of the lack of alternatives that meets all of the aforementioned effectiveness, and other advantageous criteria, the usage of natural substances in the treatment and production of textile products with antimicrobial properties, is increasing. Moreover, wool is a natural fiber which is being used in the conception and design of home textiles with the added value related with comfort and excellent thermoisolation properties. In addition, wool is an excellent environment for microorganisms and, therefore, antimicrobial treatments are sometimes required. Accordingly, the innovative strategy of this investigation considers developing new antimicrobial textiles using a natural defensive thiolated aminoacid (L-cysteine) that is found in several living organism.

The antibacterial effect of sulfhydryl agents (thiolated compounds) was previously claimed by Morris et al. ${ }^{9}$ in a study that firmly establish their reaction with the sulfhydryl groups that are located in the membrane of the Gram-positive and Gram-negative bacteria. In addition, other studies reported that not only arginine residues but also cysteine residues with free sulfhydryl groups are likely to play an important role in the expression of the antibacterial activity of the antimicrobial peptides under study. ${ }^{10}$ The mechanism by which cysteine residues participate in the antibacterial activity of these peptides remains, however, to be clearly elucidated. ${ }^{10}$

Consequently, it seems likely there is a potential in the utilization of agents which contain free sulfhydryl groups with the aim of producing antibacterial products.

Therefore, the innovative strategy of this study was to test natural agents containing free sulfhydryl groups such as L-cysteine with the aim of producing new antimicrobial wool-based textiles. The ability of L-cysteine either to bind to wool fibers trough ionic interaction or via disulfide bonds between the thiol terminals or to provide an antibacterial effect which was tested against Staphylococcus aureus and Klebsiella pneumoniae is therefore, here reported, along with the evaluation of the minimal inhibitory concentration (MIC) and of wool damage.

\section{EXPERIMENTAL}

\section{Materials}

The substrate selected to this work was a $100 \%$ Taft Wool fabric with the characteristics indicated in Table I. A previous washing was done to remove any surface residues of chemicals commonly used in the finishing of wool that could provide a false positive in relation to antimicrobial activity.

\section{Methods \\ Determination of the MIC}

The broth macrodilution method was adopted for the detection of the minimal inhibitory concentration (lowest concentration that inhibits at least 99\% of bacterial growth) of L-Cys against Staphylococcus aureus (ATCC 6538) and Klebsiella pneumoniae (ATCC11296) and was performed by following the guidelines described in the NCCLS (National Committee for Clinical Laboratory Standards, 1997, 2002) broth macrodilution standard method M07-A6 whereas the bacteria were incubated for $24 \mathrm{~h}$ at $37^{\circ} \mathrm{C}$ using Muller-Hinton Broth. These strains were selected since they are often responsible for hospital infections and also because are indicated in several standards to assess antibacterial activity of textiles.

Accordingly, the minimal inhibitory concentration was determined by serial dilution in the same type of agar (Mueller-Hinton) with concentrations ranging from $9.7 \times 10^{-2} \mathrm{~g} / \mathrm{mL}$ to $2.3 \times 10^{-5} \mathrm{~g} / \mathrm{mL}$ (Table II). The Mueller-Hinton broth was prepared from a dehydrated base following the manufacturer's instructions. The inoculum was prepared from fresh overnight broth culture and the turbidity was adjusted by raising either the amount of sterile water or microorganisms to obtain turbidity equal to the $\mathrm{n}^{\circ}$. 0.5 McFarland standard.

TABLE II

Correspondence of the Dilution Factors and Final Concentrations of the Solutions Prepared from a Stock Solution of L-Cys $800 \mathrm{mM}$

\begin{tabular}{lc}
\hline Dilutions & Concentration $(\mathrm{g} / \mathrm{mL})$ \\
\hline $1: 1$ & $9.7 \times 10^{-2}$ \\
$1: 2$ & $4.8 \times 10^{-2}$ \\
$1: 4$ & $2.4 \times 10^{-2}$ \\
$1: 8$ & $1.2 \times 10^{-2}$ \\
$1: 16$ & $6.0 \times 10^{-3}$ \\
$1: 32$ & $3.0 \times 10^{-3}$ \\
$1: 64$ & $1.5 \times 10^{-3}$ \\
$1: 128$ & $7.5 \times 10^{-4}$ \\
$1: 256$ & $3.8 \times 10^{-4}$ \\
$1: 512$ & $1.9 \times 10^{-4}$ \\
$1: 1024$ & $9.4 \times 10^{-5}$ \\
$1: 2048$ & $4.7 \times 10^{-5}$ \\
$1: 4096$ & $2.3 \times 10^{-5}$ \\
\hline
\end{tabular}


TABLE III

Growth Inhibition of the Several L-Cysteine Solutions

\begin{tabular}{lcccccccccccccc}
\hline & \multicolumn{10}{c}{ Dilutions } \\
\cline { 2 - 11 } \multicolumn{1}{c}{ Strain } & $1: 1$ & $1: 2$ & $1: 4$ & $1: 8$ & $1: 16$ & $1: 32$ & $1: 64$ & $1: 128$ & $1: 256$ & $1: 1024$ & $1: 2048$ & $1: 4096$ \\
\hline S. aureus (ATCC 29213) & I & - & - & - & - & + & + & + & + & + & + \\
K. pneumoniae (ATCC11296) & I & - & - & - & - & + & + & + & + & + & + & + \\
\hline
\end{tabular}

Caption: + turbidity (growth of inoculum); - growth inhibition; i, inconclusive due to heavy precipitation of L-Cys at the bottom of the test tube.

Totally, $1 \mathrm{~mL}$ of inoculum was added to each tube containing $1 \mathrm{~mL}$ of antimicrobial agent in the dilution range prepared from a stock solution of L-Cys 800 $\mathrm{mM}$ (Table III). The inoculated macrodilution tubes were incubated for $24 \mathrm{~h}$ at $37^{\circ} \mathrm{C}$ to assess antibacterial activity. Control tubes of the medium, broth and broth with L-Cys, were also incubated.

\section{Functionalization process}

Fresh solutions of L-Cys (Sigma-Aldrich) were prepared in a concentration range of 0.5 to $12 \%$ (w/w over the weight of the fabric) in $25 \mathrm{mM}$ acetate buffer solution $\mathrm{pH}$ 4.8. Functionalization of the wool material was performed by an exhaustion method which promotes the reaction of L-Cys with the wool using a liquor ratio of $50 / 1(\mathrm{~L} / \mathrm{kg})$. In this way, the wool fabrics were incubated for $50 \mathrm{~min}$ at $60^{\circ} \mathrm{C}$. These conditions were selected to preserve both the quality of wool and ensure the reduced form of L-Cys with the aim of endorsing an increase in the number of free sulfhydryl groups on wool to give the desired antimicrobial properties. In the end, several rinses were made followed by a soaping procedure at $40^{\circ} \mathrm{C}$ (in accordance with the recommendations of the Standard NP EN ISO 105-C06). The soaping procedure was performed over the samples treated with L-Cys prior to the assessment of antibacterial activity to give evidence of a durable functionalization effect. This soaping procedure is recommended to assess the durability of a dye on wool, to show out the fastness properties. Therefore, in the same way, this soaping procedure was performed to assess the fastness of the binding of the amino acid L-cysteine to wool fibers.

Assessment of the increase of free sulfhydryl groups on wool

The absorption of L-Cys into wool was determined using the Ellman's reagent (5,5-dithio-bis-2-nitrobenzoic acid or DTNB) ${ }^{11-13}$ to detect the presence of free sulfhydryl groups in the incubation solution before and after functionalization, which can be detected with the DTNB according to the mechanism shown in Figure 1.

Briefly, the colorimetric method was performed by measuring the absorbance of the solution at $412 \mathrm{~nm}$ before and after incubation, and by estimating the amount of sulfhydryl groups by means of calibration curves of known concentrations of L-Cys. As a result, an exhaustion rate of L-Cys was estimated trough the difference of the amount before and after functionalization. In addition, the absorption rate of L-Cys was accurate by measuring the color changes directly on the fabrics. For this purpose, a modification was made to the Ellman's method described in the literature. ${ }^{11-13}$ Briefly, a dyeing procedure with the Ellman's reagent, in this case used as a dye over the wool samples, was performed by using $4 \%$ $(\mathrm{w} / \mathrm{w}$ owf) of DTNB reagent, a liquor ratio of $50 / 1$ $(\mathrm{mL} / \mathrm{g})$ for $60 \mathrm{~min}$ at $60^{\circ} \mathrm{C}$. Subsequently, the relative color strength $(K / S)$ was measured on a reflectance measuring apparatus Spectraflash 300 Datacolor (LAV/Spec. Incl., d/8, D65/10 ${ }^{\circ}$ ) at $410 \mathrm{~nm}$ to estimate the free sulfydryl groups and assess the immobilization of L-Cys on wool. $K / S$ values were established according to the Kubelka-Munk eq. (1), where $K$ and $S$ stand for the absorption and scattering coefficients and $R$ stands for the reflectance value, respectively, ${ }^{14}$ :

$$
\frac{K}{S}=\frac{(1-R)^{2}}{2 R}
$$

According to this adapted method it is expected that the functionalized samples would show an increase in the yellow color intensity if there is a greater amount of free sulfydryl groups in relation to the control and, consequently, a higher value for $K / S$.

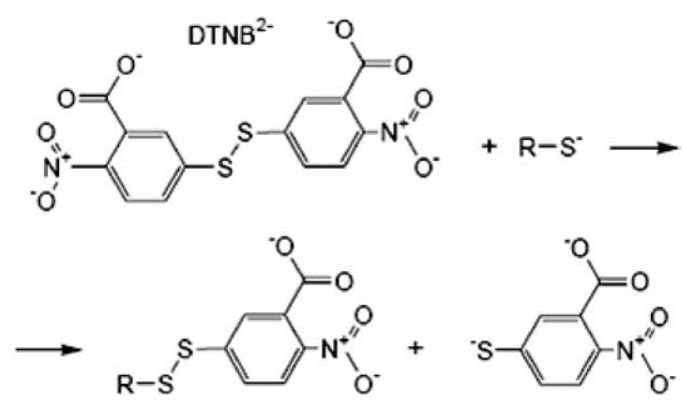

Figure 1 Reaction mechanism of the sulfhydryl groups with the DTNB. 
TABLE IV

Indirect Measurement of the Sulfhydryl'S Groups Content: Values for the $K / S$ and for the Exhaustion Rates

\begin{tabular}{ccc}
\hline $\begin{array}{c}\text { L-cysteine } \\
\text { conc. }(\% \mathrm{w} / \mathrm{w})\end{array}$ & K/S & Exhaustion rates $(\%)$ \\
\hline 1 & $0.59 \pm 0.04$ & $33.63 \pm 0.68$ \\
2 & $0.60 \pm 0.06$ & $36.00 \pm 0.52$ \\
3 & $0.65 \pm 0.04$ & $35.94 \pm 0.60$ \\
4 & $0.79 \pm 0.04$ & $36.20 \pm 0.68$ \\
5 & $1.20 \pm 0.05$ & $38.00 \pm 0.68$ \\
6 & $1.32 \pm 0.04$ & $36.98 \pm 0.68$ \\
7 & $1.38 \pm 0.04$ & $38.12 \pm 0.68$ \\
8 & $1.34 \pm 0.04$ & $37.68 \pm 0.68$ \\
\hline
\end{tabular}

Assessment of antibacterial activity of wool

The evaluation of the antibacterial activity of the functionalized samples was carried out according to the Japanese Industrial Standard JIS L 1902-2002, widely used for the antibacterial assessment of textiles. The microorganisms included Gram-positive and Gram-negative bacteria, Staphylococcus aureus (ATCC ATCC 6538) and Klebsiella pneumoniae (ATCC 11296), respectively. After preparation of inoculum and bacterial culture, as described in this standard, both functionalized samples and control were tested. For the qualitative method, the Petri dishes were placed in a heater upside down, where they were incubated for a period of $24-48 \mathrm{~h}$ at a temperature of $37^{\circ} \mathrm{C}$. Assays were run in triplicate.

In addition to qualitative method, quantitative tests were also used to determine more accurately the percentage of microbial growth inhibition. The quantitative determination of antimicrobial activity was based on the Absorption method JIS L 1902 : 2002 protocol and all assays where performed in duplicate and repeated three times.

\section{Evaluation of cytotoxicity}

The release of toxical substances from a textile product with skin contact is the precondition for the formation of skin irritations. Therefore, examination on cytotoxicity permits the evaluation of the danger potential for a skin irritation.

In this examination, a perspiration extract of the test material at high L-Cys concentration $(6 \% \mathrm{w} / \mathrm{w}$ owf) under which total inhibition of bacterial growth was achieved, was produced according to E DIN EN ISO 10993-5. Typically, the test material was incubated with an acid perspiration solution according to standard DIN EN ISO 105-E04 for $24 \mathrm{~h}$ at $37^{\circ} \mathrm{C}$ under slight shaking. This so-called perspiration extract was set up to a $\mathrm{pH}$ value of 7.3-7.4 with sodium hydroxide and sterile filtrated. Connective tissue cells L 929 [ATCC No. CCL1, NCTC clone 929 L (DSMZ)], from the connective tissue of the mouse) were treated for $68-72 \mathrm{~h}$ with this solution in a dilution level of $33.3 \%-4.4 \%$. Test groups included solvent control (Phosphate buffered solution (PBS) diluted in culture medium corresponding to the test material), positive control (DMSO $(5 \%)$ in culture medium), negative control (culture medium) and test material (Concentrations of the test material in culture medium: $4.4 \%, 6.6 \%, 9.9 \%, 14.8 \%, 22.2 \%$, and $33.3 \%)$. A positive and negative control was carried out along the experiment, to confirm the validity of the test system.

After the incubation period the protein content of the cultures (Culture medium: DMEM with 10\% FBS) was compared with the protein content of the controls and from that the cell growth was determined in the presence of the test material. Modified proliferation and partition rates of the cells are shown (growth inhibition test) in the presence of cell-toxic substances.

\section{Assessment of wool damage}

The eventual damage caused on wool fabrics after functionalization with L-Cys was evaluated with the standard method of resistance to abrasion (Martindale Standard method EN IS0 12947-2). In this method, samples treated with $6 \%(\mathrm{w} / \mathrm{w}$ owf) were tested under a weight of $9 \mathrm{kPa}$ and run until the rupture of two yarns. Significant decreases in the resistance to abrasion would reveal important fiber damage. In addition, SEM analysis was used to evaluate any surface damage on the same functionalized wool samples, in comparison to a control. Samples were observed with a scanning electron microscope (HITACHI S2700 Electron Microscope and EMITECH-K550 gold evaporator).

\section{RESULTS AND DISCUSSION}

\section{Determination of the minimal inhibitory concentration}

The broth macrodilution method was used for the detection of the minimal inhibitory concentration of L-Cys against Staphylococcus aureus (ATCC 6538) and Klebsiella pneumoniae (ATCC 11296) and was performed by following the guidelines described in the NCCLS M07-A6 standard method. For the higher concentrations of L-Cys $\left(9.7 \times 10^{-2} \mathrm{~g} / \mathrm{mL}\right)$ the possible oxidation of the amino acid with the consequent formation of crosslinking via disulfide bonds may have led to the formation of aggregates that precipitates in aqueous solutions at the bottom of the tubes, causing a very difficult reading of the results. For less concentrate solutions, however, that effect was not observed and the estimated minimal inhibitory concentrations of L-cysteine against $S$. aureus and $K$. pneumoniae were found to meet within the range 


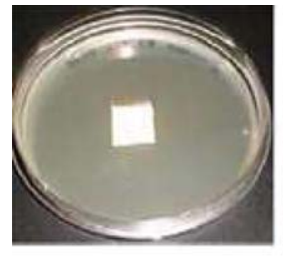

With textile

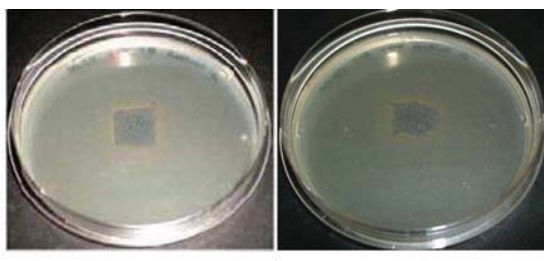

S. aureus
K. pneumoniae
Figure 2 Antibacterial assay: inhibition effect of the functionalized samples $(1 \% \mathrm{w} / \mathrm{w})$ against Staphylococcus aureus and Klebsiella pneumoniae. [Color figure can be viewed in the online issue, which is available at wileyonlinelibrary. com.]

of $6.0 \times 10^{-3}-4.8 \times 10^{-2} \mathrm{~g} / \mathrm{mL}$ [MIC $0.6 \%(\mathrm{w} / \mathrm{v})$ ] and $6.0 \times 10^{-3}-4.8 \times 10^{-2} \mathrm{~g} / \mathrm{mL}$ [MIC $0.6 \%(\mathrm{w} /$ $\mathrm{v})]$, respectively. In addition, the results of the various controls showed a normal growth on the medium indicating the viability of the strains.

These MIC values are significantly higher in comparison with others obtained for example for chitosan $(0.05 \%(\mathrm{w} / \mathrm{v})$ against S. aureus and K. pneumonia) and for triclosan $\left(5.3 \times 10^{-6} \%(\mathrm{w} / \mathrm{v})\right.$ against $S$. aureus). ${ }^{15,16}$ However, the ability of L-Cys for crosslinking once in an aqueous solution might have an important role in these results since a higher concentration should be required to obtain the sulfydryl groups in a free form with the consequent antibacterial activity. However, once in the wool, the immobilization might require less concentrate L-Cys solutions to give wool the desired antibacterial activity, as discussed below.

\section{Assessment of the increase of free sulfhydryl and related antimicrobial and cytotoxic properties}

Figure 1 shows the mechanism of reaction between Ellman's reagent and the sulfhydryl groups. The DTNB reagent has a specific affinity for free sulfhydryl groups in solution and in wool fiber. Thus, a deeper color shows the existence of a greater number of free sulfhydryl groups as a result of absorption and reaction of Lcysteine with wool fibers through the linkage between amino and carboxylic groups both of wool and L-cysteine. The increase in color intensity, measured on a spectrophotometer at $410 \mathrm{~nm}$, clearly point out the presence of a greater number of free sulfhydryl groups in the functionalized wool fabrics for all L-Cys concentrations (Table IV), indicating that the major sulfhydryl groups are on their free form once immobilized on the wool material, suggesting an absorption mechanism via ionic interaction between the amino and carboxylic groups of L-cysteine and wool. However, the absorption mechanism via disulfide bonds between the thiol terminals of cysteine residues of wool and thiol terminals of the amino acid L-cysteine is expected as well, depending on the extension of the ionization of these groups which is strongly correlated with a $\mathrm{pH}$ value of the incubation solution above $8 .{ }^{17}$ In addition, the exhaustion rates were more or less the same for all the concentrations used highlighting a good diffusion process of L-Cys whichever the concentration in the range used (Table IV). The results also showed a higher content of L-Cys above $4 \%(\mathrm{w} / \mathrm{w})$.

On the other hand, the results of the qualitative antibacterial assays against S. aureus and K. pneumoniea revealed an antibacterial activity of the wool samples that were functionalized with L-Cys even at the lower concentrations of L-Cys $(1 \% \mathrm{w} / \mathrm{w})$ (Figure 2), showing that the lowest values of K/S that were used to indirectly measure the free sulfhydryl groups on the textiles are related with an antimicrobial activity. Accordingly, Table $\mathrm{V}$ shows the qualitative results for the antibacterial effect whereas the inhibition zone is located under the textile. This type of inhibition under the samples always happens whenever the biocide agent does not possess the ability to migrate and, therefore, inhibition is restricted to the textile and to the zones that are in close contact with the antimicrobial textile. In addition, the percentage of growth inhibition against one of the strains (S. aureus, since the MIC values were the same for the both strains), caused by the samples functionalized with L-Cys concentrations in the range of $0.5-12 \%(\mathrm{w} / \mathrm{w}$ over the weight of the fabric), calculated according with the JIS L 1902 - Absorption method, is presented in Figure 3. The results show evidence of $40 \%$ inhibition for L-Cys concentrations of $1 \%$ and a total inhibition (more than $99 \%$ ) above $6 \%$. In addition, the $\mathrm{MIC}_{50}$ is obtained in samples with $5 \%$ of L-Cys, approximately.

In general, the incorporation of the majority of the well known and commercial biocides in the textiles reduces the bioactivity substantially and limits availability, compelling the raised concentrations to be used in the functionalization process. ${ }^{1}$ In contrary, in the present study, the L-Cys concentration of $6 \%(\mathrm{w} /$ $\mathrm{w}$, owf) which enable growth inhibition above $99 \%$ is equivalent to $1.2 \times 10^{-3} \mathrm{~g} / \mathrm{mL}$, clearly much lower than the $6.0 \times 10^{-3} \mathrm{~g} / \mathrm{mL}$ found out for the

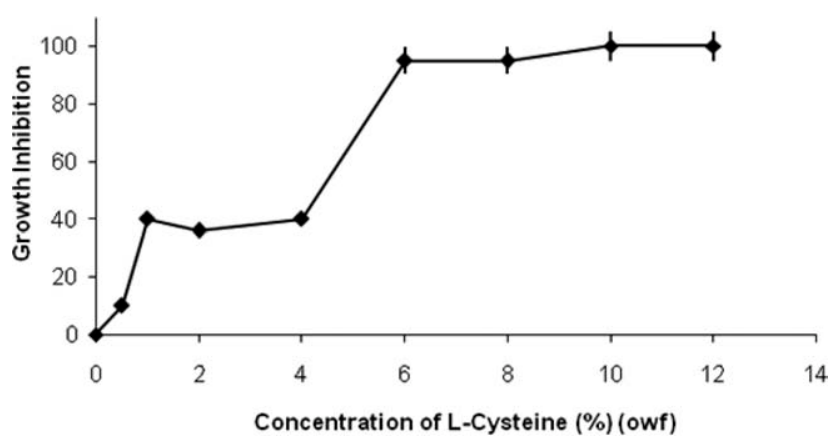

Figure 3 Growth inhibition percentage against S. aureus, of the several L-cysteine concentrations used. 


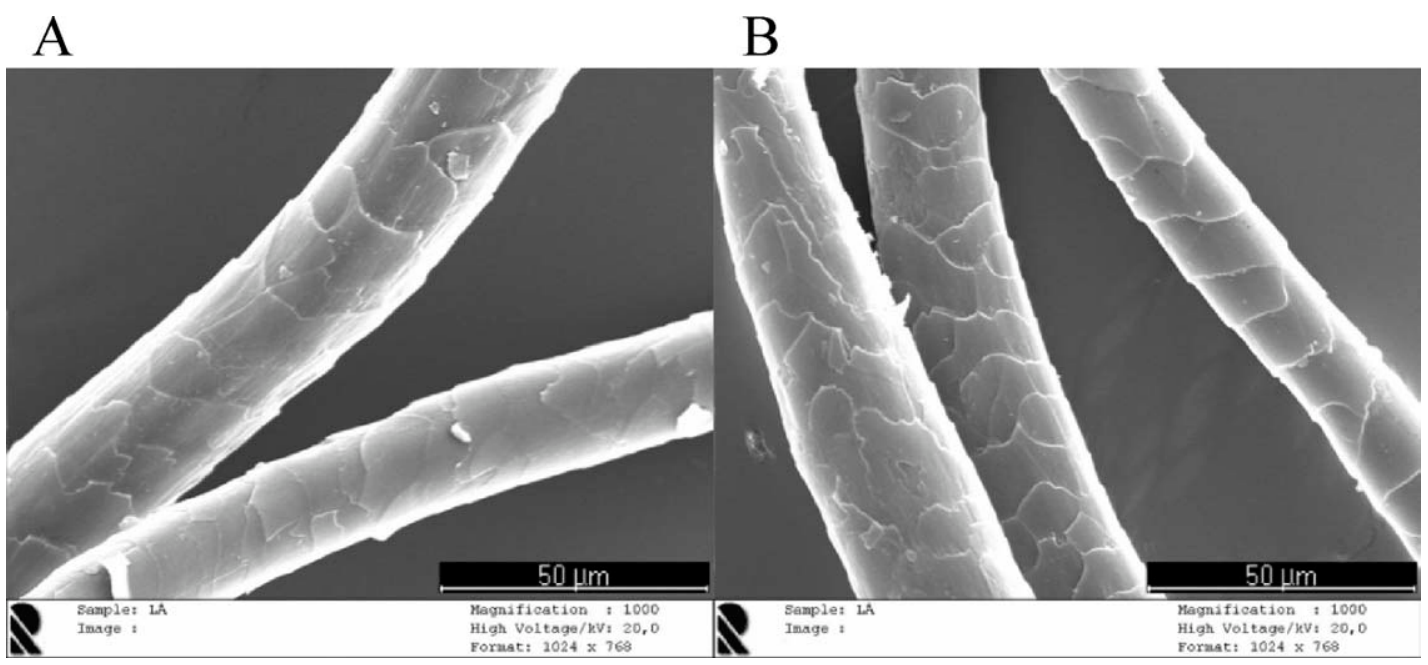

Figure 4 Surface morphology of the control (A) and functionalized wool fibers (B).

antimicrobial activity of L-Cys in solution, against the same strain (Klebsiella pneumoniae), which means that less concentrations are required to obtain antimicrobial activity over wool. These results also provide important guidelines to develop new strategies and applications whereas L-Cys could play an important role in the control of microbial growth on textiles, as for example as an additive for detergents to be used in domestic or industrial laundry.

The antimicrobial effect that L-Cys provides to the wool is in accordance with the results found out for the antimicrobial activity of thiosulfinates which proposes that the antimicrobial effect results from the interaction of sulfur groups present in thiosulfinates with the sulfhydryl groups of the proteins and enzymes that are essential to the survival of microorganisms, leading to growth inhibition and death. ${ }^{17,18}$ According to these studies, thiosulfinate compounds have the ability to inhibit microbial growth in Staphylococcus aureus, Salmonella typhimurium, Bacillus cereus, Clostridium botulinum, and Candida utilis among others. It also appears that these thiolated molecules have a high structural diversity and a broad spectrum of activity that includes Gram-positive and Gram-negative bacteria, fungi, and in particular cases, some viruses with the added advantage of not displaying cytotoxicity to mammalian cells, as described elsewhere. ${ }^{19,20}$ Therefore, the functionalization of the wool fabrics with L-cysteine gave an excellent and durable antibacterial activity even for the lowest concentrations tested $(1 \% \mathrm{w} / \mathrm{w}$, owf $)$, being a promising new approach that can also diminish bacterial resistance.

Furthermore, as per Borenfreund and Borrero, ${ }^{21}$ the protein content of the cell culture assays can be used as reference for the growth of the L 929 mouse fibroblasts respectively, for the growth inhibition in the presence of cell-toxic substances. A growth inhibition of more than $30 \%$ in comparison with the solvent control is assessed as a clear cell-toxic effect. ${ }^{21}$ This value is usually reached at the highest extraction step of $33.3 \%$ of the perspiration extract described in the E DIN EN ISO 10993-5.

The perspiration extract of the test material (functionalized wool with $6 \%(\mathrm{w} / \mathrm{w})$ of L-Cys) showed a growth inhibition of $25 \%$ in the cytotoxicity test under the mentioned conditions. In accordance to this, it can be concluded that no cytotoxic substances are released during the assigned use of these test materials, which can lead to irritations with skin contact.

\section{Assessment of wool damage}

Finally, with regards with the resistance to abrasion tests performed over the control and wool samples functionalized with $6 \%(\mathrm{w} / \mathrm{w})$ the results are as follows:

Totally, 34,000 \pm 3000 cycles for the control and $37,500 \pm 7500$ for the functionalized wool. Although it appears that the functionalized wool present a higher resistance, the deviation to the mean is also higher. In this way, it may happen that in the limit a sample of antimicrobial wool may present a resistance of 30,000 cycles in comparison with the control in which the minimum resistance can be up to 31,000 cycles. This represents less than $4 \%$ of loss of resistance by the action of L-Cys under the evaluated conditions meaning that there is no significant loss of resistance of wool, as described elsewhere. ${ }^{22}$

The surface morphology of the biofunctionalized wool was observed against a control with a scanning electron microscope. Images indicated in Figure 4 clearly show no evidence of surface alteration or damage. Therefore, the functionalization of wool with L-Cys even at high concentrations $(6 \%)$ does not alters the surface morphology of wool fibers. 


\section{CONCLUSION}

This work describes a novel method to give antibacterial activity to wool fibers using a nontoxic agent-L-cysteine, a natural product never studied before as a potential antimicrobial agent for textiles, which can grant antibacterial properties without cytotoxicity. The major advantages of this method in comparison with others that are commonly used to give antimicrobial properties onto textile materials are the nontoxicity both to the potential users and to the environment, the high bioavailability of L-cysteine once immobilized on wool, representing lower loadings of biocides to obtain antimicrobial activity, the durability, and the possibility of being carried out in a simple process with short reaction time and mild temperature. In addition, because L-cysteine is part of several living organisms it is not expected to cause bacterial resistance. This is totally new and will able to open new applications for wool, in particular, in health-care sector. This novel process may also open new avenues for the biomimetic design of antimicrobial materials for medical applications.

The authors acknowledge the grammar revision provided by João M. Martins.

\section{References}

1. Gao, Y.; Cranston, R. Text Res J 2008, 78, 60.

2. Ramachandran, T., et al. IE (I) J - TX 2004, 84, 42.

3. Purwar, R.; Joshi, M. AATCC Rev 2004, 4, 22.

4. Ammayappan, L.; Jeyakodi, M. J Fibers Polym 2009, 10, 161.

5. Han, S.; Yang, Y. Dyes Pigments 2005, 64, 157.

6. Quin, L.; Sun, G. J Appl Polym Sci 2004, 91, 2588.

7. Elsner, P. Biofunct Text Skin 2006, 33, 35.

8. Askew, P. D. Chem Today 2009, 27-1, 16.

9. Morris, S.; Walsh, C.; Hansen, Q. N. J Biol Chem 1984, 259, 13590.

10. Yomogida, S.; Nagaoka, I.; Yamashita, T. Infect Immun 1995, 63, 2344.

11. Ellman, G.L. Arch Biochem Biophys 1959, 82, 70.

12. Riddles, P.W., et. al. Anal Biochem 1979, 94, 75.

13. Riddles, P.W., et al. Method Enzymol 1983, 91, 49.

14. Pandiyaraj, K. N.; Selvarajan, V. J Mater Process Technol 2008, 199, 130.

15. Kim, Y. H.; Nam, C. W.; Choi, J. W.; Jang, J. J. Appl Polym Sci 2003, 88, 1567.

16. Cottell, A.; Denyer, S. P.; Hanlon, G. W.; Ochs, D.; Maillard, J.-Y. J Hosp Infect 2009, 72, 71.

17. Kyung, K. H.; Lee, Y. C. Food Rev Int 2001, 17, 183.

18. Wilson, E. A.; Adams, B. D. Nutr Food Sci 2007, 37, 178.

19. Dimarcq, J. L.; Bulet, P.; Hetru, J.; Hoffmann, C. Biopolymers 1998, 47, 465.

20. Ganz, T. Biologies 2004, 327, 539.

21. Borenfreund, E.; Borrero, O. Cell Biol Toxicol 1984, 1, 55.

22. Gouveia, I. C.; Antunes, L. C.; Gomes, A. P. J Text Institute 2011, 102, 203. 